\title{
Driving Point Impedance for a Linear Array of Half-width Leaky-wave Antennas
}

\author{
Michael Corwin*, Leo Kempel, Henry Griffith, and Steve Schneider \\ Air Force Research Laboratory/ Sensors Directorate (AFRL/SND) \\ Wright-Patterson AFB, Dayton, OH 45433
}

\begin{abstract}
Printed leaky-wave antennas offer the potential for a low-profile, wide-bandwidth antenna element that can be arrayed if desired. Microstrip leaky-wave antennas rely on the suppression of the familiar $\mathrm{EH}_{0}$ mode and the propagation of the radiating $\mathrm{EH}_{1}$ mode. It is well-known that above a critical frequency, this leaky-wave will propagate with little attenuation and that the phase difference between the two radiating edges of the microstrip leads to radiation. The driving point impedance for a given leakywave antenna
\end{abstract}

Keywords: Leaky-wave Antenna; Linear Array; Impedance

\section{Introduction}

Microstrip leaky-wave antennas offer the potential for a low-profile antenna with greater bandwidth than microstrip patch antennas. This is principally due to the fact that the radiation mechanism is attributed to a traveling-wave as compared to the standing-wave that is responsible for radiation by a microstrip patch antenna. The fundamental mode of a microstrip line, the so-called $\mathrm{EH}_{0}$ mode, is not a radiating mode (hence the popularity of microstrip transmission lines). The electric and magnetic fields for this mode are shown in Figure 1.

Higher-order modes must be preferentially excited to realize a radiating traveling wave structure. The first higher-order mode, the $\mathrm{EH}_{1}$ mode, is one such radiating mode. This mode (shown in Figure 2) exhibits electric field odd symmetry about the axial centerline of the antenna as compared to the even symmetry of the $\mathrm{EH}_{0}$ mode.

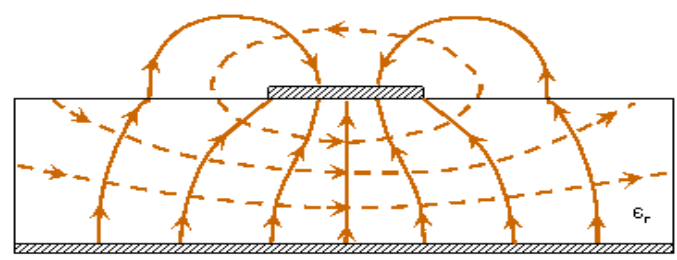

Figure 1. Field diagram for the $\mathbf{E H}_{0}$ mode (E-field = solid, H-field $=$ dashed $)$.

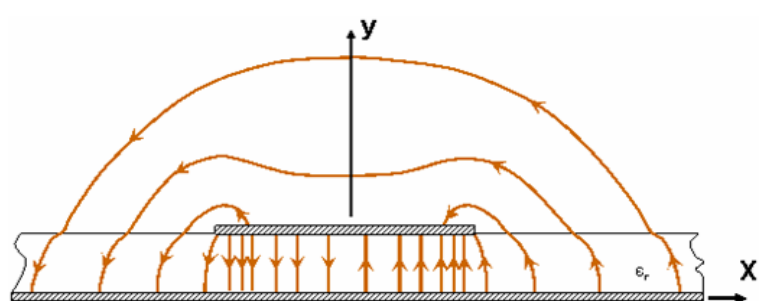

Figure 2. Field diagram for the $\mathrm{EH}_{1}$ mode $(\mathrm{E}$ field = solid, $\mathrm{H}$-field $=$ dashed).

A common method for estimating the radiation bandwidth of an $\mathrm{EH}_{1}$-mode antenna is based on a waveguide model [1-3]. The bandwidth of such an antenna is determined by the cut-off frequency for the $\mathrm{EH}_{1}$ mode and the frequency where the phase constant equals $\mathrm{k}_{0}$ (e.g. above this frequency, the bound mode propagates rather than the leaky-wave). This frequency range is given by

$$
\mathrm{f}_{\mathrm{c}}=\frac{15}{\mathrm{w}_{\text {eff }} \sqrt{\varepsilon_{\mathrm{r}}}}<\mathrm{f}<\frac{\mathrm{f}_{\mathrm{c}} \sqrt{\varepsilon_{\mathrm{r}}}}{\sqrt{\varepsilon_{\mathrm{r}}-1}}=\frac{15}{\mathrm{w}_{\text {eff }} \sqrt{\varepsilon_{\mathrm{r}}-1}}
$$

where the zero-thickness effective width of the microstrip (in cm) is given by Wheeler [4-6]

$$
\mathrm{w}_{\text {eff }}=\mathrm{h}\left\{\frac{\mathrm{w}}{\mathrm{h}}+\frac{2}{\pi} \ln \left[2 \pi \mathrm{e}\left(\frac{\mathrm{w}}{2 \mathrm{~h}}+0.92\right)\right]\right\}
$$

Operation of the microstrip in the range of frequencies specified by (1) will allow the propagation of the $\mathrm{EH}_{1}$ leaky-wave mode; however, the fundamental $\mathrm{EH}_{0}$ will also propagate and depending on the feeding 
structure, this fundamental mode may dominate. One significant challenge in realizing a printed leakywave antenna is the suppression of the fundamental mode in preference to the desired $\mathrm{EH}_{1}$ mode. Various means of preferentially exciting the $\mathrm{EH}_{1}$ mode have been investigated (see for example [3] and the references contained within that manuscript). Another means of preferentially exciting the $\mathrm{EH}_{1}$ mode is to suppress the $\mathrm{EH}_{0}$ mode. This was accomplished using small slots in the microstrip structure near the feed region by Mentzel [7] and an asymmetric microstrip feed (including a quarter wave transformer to improve the match to a $50 \Omega$ port). An alternative method, utilizing a physical grounding structure along the length of the antenna, has recently been investigated by the Air Force Research Laboratory [8]. The major advantage of such an antenna is the reduced width as compared to other leaky-wave microstrip antennas and the relatively simple feed structure. Recently, the FD-TD method was used to study this antenna [9] in addition to experiments. An alternative method is the finite element method that has the advantage of being able to model more complex geometrical aperture arrangements.

\section{Finite Element-Boundary Integral Model of the Half-width Antenna}

The FE-BI method has been extensively reported upon in the literature and is described in at least three popular text books on finite element methods for high frequency electromagnetics [10-12]. The FE-BI equations for a total electric field formulation may be written as

$$
\begin{aligned}
& \int_{\mathrm{V}}\left[\nabla \times \mathbf{W}_{\mathrm{i}} \cdot \overline{\bar{\mu}}_{\mathrm{r}}^{-1} \cdot \nabla \times \mathbf{W}_{\mathrm{j}}\right] \mathrm{dV}-\mathrm{k}_{0}^{2} \int_{\mathrm{V}}\left[\mathbf{W}_{\mathrm{i}} \cdot \overline{\bar{\varepsilon}}_{\mathrm{r}} \cdot \mathbf{W}_{\mathrm{j}}\right] \mathrm{dV}+\mathrm{jk}_{0} \int_{\mathrm{S}_{\mathrm{R}}}\left[\frac{\left(\hat{\mathrm{n}}_{\mathrm{R}} \times \mathbf{W}_{\mathrm{i}}\right) \cdot\left(\hat{\mathrm{n}}_{\mathrm{R}} \times \mathbf{W}_{\mathrm{j}}\right)}{\mathrm{R}_{\mathrm{e}}}\right] \mathrm{d} \mathrm{S} \\
& -\mathrm{k}_{0}^{2} \int_{\mathrm{S}_{\mathrm{a}}} \int_{\mathrm{S}_{\mathrm{a}}}\left[\mathbf{W}_{\mathrm{i}} \cdot \hat{\mathrm{z}} \times \overline{\overline{\mathrm{G}}}_{\mathrm{e} 2} \times \hat{\mathrm{z}} \cdot \mathbf{W}_{\mathrm{j}}\right] \mathrm{d} \mathrm{S}^{\prime} \mathrm{d} S=\mathrm{f}_{\mathrm{i}}^{\mathrm{int}}+\mathrm{f}_{\mathrm{i}}^{\text {ext }}
\end{aligned}
$$

where the first term is associated with the curl of the basis function (the magnetic field), the second term is associated with the basis function itself (the electric field), the third term is necessary to account for an resistive transition conditions if present, and the last term on the left-hand side is the boundary integral term (involving a second kind electric field dyadic Green's function). As shown, the material parameters are represented as tensors $\left(\overline{\bar{\varepsilon}}_{\mathrm{r}}, \overline{\bar{\mu}}_{\mathrm{r}}\right)$ while the basis and test functions are represented by $\left(\mathbf{W}_{\mathrm{j}}\right)$ and $\left(\mathbf{W}_{\mathrm{i}}\right)$, respectively. For this work, brick elements are used along with a half-space dyadic Green's function

$$
\overline{\overline{\mathrm{G}}}_{\mathrm{e} 2}\left(\mathrm{x}, \mathrm{y} \mid \mathrm{x}^{\prime}, \mathrm{y}^{\prime}\right)=\left[\overline{\overline{\mathrm{I}}}+\frac{\nabla \nabla}{\mathrm{k}_{0}^{2}}\right] \frac{\mathrm{e}^{+\mathrm{jkR}}}{2 \pi \mathrm{R}}
$$

where $\overline{\bar{I}}=\hat{x} \hat{x}+\hat{y} \hat{y}+\hat{z} \hat{z}$ and $R=\sqrt{\left(x-x^{\prime}\right)^{2}+\left(y-y^{\prime}\right)^{2}+\left(z-z^{\prime}\right)^{2}}$.

\section{Candidate Half-width Leaky-wave Antenna}

As an example, consider a leaky-wave antenna printed on Duroid 5870 (31 mils thick, $\left.\varepsilon_{\mathrm{r}}=2.33, \tan \delta=0.0005\right)$. The full-width strip width is $15 \mathrm{~mm}$ while the half-width strip width is 7.5 $\mathrm{mm}$. The strip is $190 \mathrm{~mm}$ long and terminated with a lumped $50 \Omega$ load placed midway between the shorted edge and the free edge of the antenna. This load placement was determined, using TRM and confirmed using numerical techniques, as suitable for wide bandwidth termination of the antenna [14].

The half-width antenna utilizes a physical electric conducting wall to suppress the fundamental mode. This is accomplished in the finite element model with an infinitesimally thin perfect conducting wall while in the actual antenna constructed in the RASCAL facility at AFRL, the wall is formed with closely spaced periodic conducting pins. The feed is a probe feed placed half-way along the width of the half-width antenna (consistent with the placement of the co-planar microstrip feeds in [13]) and slightly inset. According to (1), the leaky-wave region of the antennas is given by $6.55 \mathrm{GHz} \leq \mathrm{f} \leq 8.67 \mathrm{GHz}$. Below this range of frequency, the $\mathrm{EH}_{1}$ mode is in cut-off while above that range, the bound mode is dominant. In [9], the data generated from both the FDTD and transverse resonance models indicate that the attenuation of the propagating wave is quite small in the leaky-wave band and hence, a relatively short 
antenna (as is the one in this work) will have a significant backward traveling leaky-wave without a suitable termination.

\section{Results for a Linear Array}

For this study, the driving point impedance is computed for a single terminated element, three terminated elements, and five terminated elements. The apertures for these are shown in Figure 3. In this, the substrate is represented by blue, the microstrip structure by red, the shorting wall by white, the feed by a black dot, and the load by a green dot. As can be seen, the physical aperture dimensions were kept constant and the antenna was centered in the $x-y$ plane.

To establish convergence of the geometrical/electrical representation of the antenna, the voltage standing wave ratio (VSWR) assuming a $50 \mathrm{Ohm}$ port and a $50 \mathrm{Ohm}$ termination impedance were calculated. The mesh density along the length was set at $0.125 \mathrm{~cm}$ while the density along the width of a single antenna (See Figure 3) was $0.25 \mathrm{~cm}$, $0.125 \mathrm{~cm}$, and $0.0625 \mathrm{~cm}$. Since the antenna is assumed to be reasonably well-matched if meshed sufficiently, the longitudinal field variation is expected to be fairly small (e.g. little standing wave). Assuming a quasi-TEM wavelength, the longitudinal sample rate is approximately $\lambda_{\mathrm{g}} / 20$. The lateral field variation is
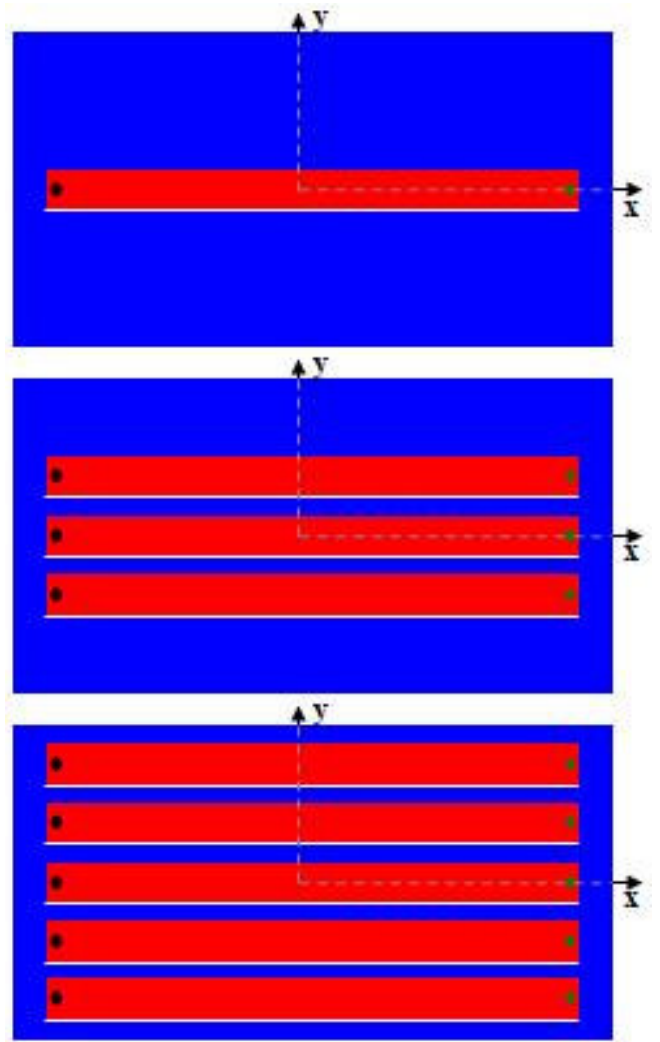

Figure 3. Three linear array geometries. expected to be one quarter of a sine function (see [3,14] for example), a higher sample rate may be necessary. Figure 4 illustrates the comparison showing that a lateral sample rate of $\lambda_{\mathrm{g}} / 40$ is sufficient for understanding the local behavior of these antennas.

The input impedance is now compared for the three cases illustrated in Figure 3. It is expected that the input impedance will be impacted by the presence of both driven and passive near-by antennas due to the reasonably strong coupling between elements attributed to the leaky-wave itself. The results are shown in Figure 5 where the impedance of the center element for a $1 \times 1$ and a $5 \times 1$ array are shown in real and complex form from 5-9 GHz. As can be seen, both the resistance and the reactance exhibit variation due to the presence of driven near-by elements. Further

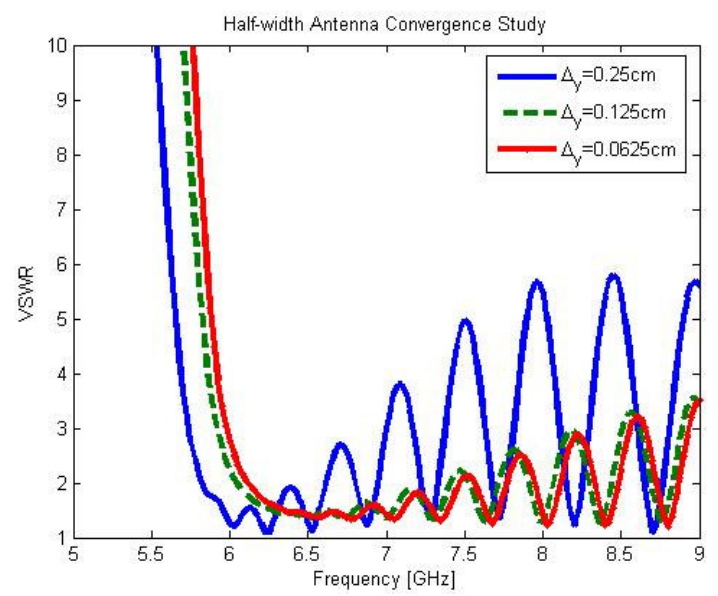

Figure 4. VSWR convergence study. examples involving the effect of passive elements will be shown at the meeting.

\section{Conclusions}

In this paper, the hybrid finite element-boundary integral method was used to investigate the properties of a leaky-wave antenna. The antenna under study was the half-width antenna recently introduced by Radcliffe et al. [8]. In this, a physical structure (realized via periodic shorting pins) is used to suppress the fundamental - even - mode. Probe feeding was used in the model since this antenna does not require a sophisticated feed structure as was needed in [13] to suppress the fundamental mode.

A converged representation of a single HWLW element was found by increasing the lateral sample rate. That antenna model was used to compare the diving-point impedance of a single terminated element with a small array of terminated HWLW antennas. Fairly significant differences existed in the driving-point 
impedance implies a fairly strong mutual impedance between elements; presumably attributed to the leakywaves excited in each antenna element. Hence, there is a clear need to compensate for mutual coupling in the design of such arrays and that pattern multiplication array beam synthesis will in general not be sufficient. Further results will be presented at the meeting.

\section{References}

1. C.S. Lee, V. Nalbandian, and F. Schwering, "Planar dual-band microstrip antenna," IEEE Trans. Antenna. Propagat., 43, pp. 892-895, Aug. 1995.

2. C.S. Lee and V. Nalbandian, "Planar leaky-wave microstrip antenna," Proc. IEEE AP-S Int. Sypm. Dig., pp. 1126-1129, 1997.

3. W. Hong, T-L Chen, C-Y Chang, J-W Sheen, and Y-D Lin, "Broadband tapered microstrip leakywave antenna," IEEE Trans. Antennas Propagat.,

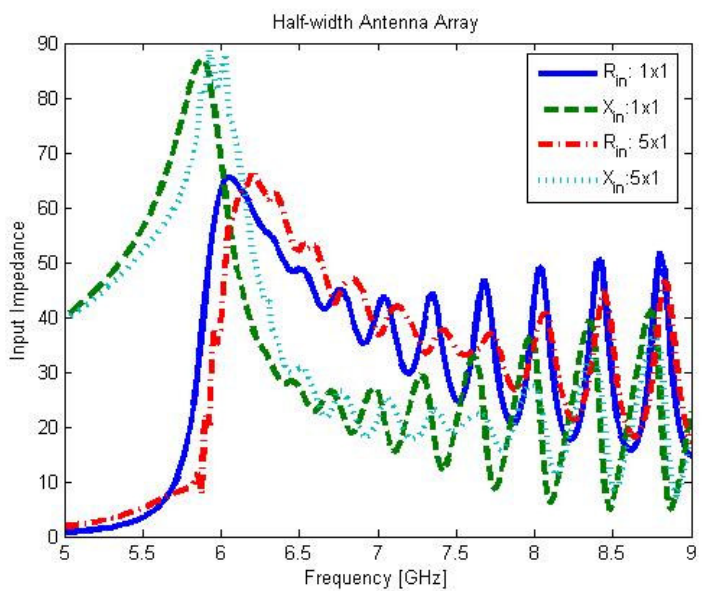

Figure 5. Comparison of the driving-point impedance for the center element of a 1x1 and a $5 \times 5$ array. 51, pp. 1922-1928, Aug. 2003.

4. H.A.Wheeler, "Transmission line properties of parallel wide strips by conformal mapping approximation," IEEE Trans. Microwave Theory Tech., 12, pp. 280-289, 1964.

5. H.A. Wheeler, "Transmission line properties of parallel strips separated by a dielectric sheet," IEEE Trans. Microwave Theory Tech., 13, pp. 172-185, 1965.

6. I. Wolff, "The waveguide model for the analysis of microstrip discontinuities," Chpt. 7 in Numerical Techniques for Microwave and Millimeter-wave Passive Structures, T. Itoh (Ed.), Wiley Interscience: New York, 1989.

7. W. Menzel, "A New Traveling-Wave Antenna in Microstrip", Archiv fur Elektronik und Ubertragungstechnik (AEU), Band 33, Heft 4:137-140, April 1979.

8. J.S. Radcliffe, G.A. Thiele, and G. Zelinski, "A microstrip leaky wave antenna and its properties, $26^{\text {th }}$ Antenna Measurement Tech. Assoc. Meeting, St. Mountain, GA, Oct. 2004.

9. G.M. Zelinski, M.L. Hastriter, M.J. Havrilla, J.S. Radcliffe, A.J. Terzuoli, and G.A. Thiele, "FDTD analysis of a new leaky traveling wave antenna," submitted to the 2005 IEEE/ACES Intl. Conf., Honolulu, Hawaii, Apr. 2005.

10. J.L. Volakis, A. Chatterjee, and L.C. Kempel, Finite Element Methods for Electromagnetics, IEEE Press: Piscataway, N.J., 1998.

11. M. Salazar-Palma, T. Sarkar, L-E Garcia-Castillo, T. Roy, and A. Djordjevic, Iterative and SelfAdaptive Finite-Elements in Electromagnetic Modeling, Artech House: Norwood, MA, 1998.

12. J-M Jin, The Finite Element Method in Electromagnetics, $2^{\text {nd }}$ Ed., Wiley: New York, 2002.

13. Y-D Lin, J-W Sheen, and C-K. C. Tzuang, "Analysis and design of feeding structures for microstrip leaky wave antennas," IEEE Trans. Microwave Theory Tech., 44, pp. 1540-1547, Sept. 1996.

14. D. Killips, M. Corwin, L. Kempel, and S. Schneider, "Termination of Half-width Leaky-wave Antennas," IEEE Antennas Wireless Propagat. Letters, submitted for publication, 2006.

15. D. Killips, J. Radcliffe, L. Kempel, and S. Schneider, "Radiation by a Linear Array of Half-Width Leaky-Wave Antennas," ACES Journal, submitted for publication, 2005. 